\title{
Diagnostik und Therapie der Psoriasisarthritis
}

\section{Kneitz ${ }^{1}$ \\ M. Goebeler ${ }^{2}$ \\ H.-P. Tony ${ }^{1}$}

\section{Diagnostics and Treatment of Psoriatric Arthritis}

\section{Zusammenfassung}

Die Diagnose einer Psoriasisarthritis (PsA) wird bei vielen Psoriasispatienten häufig erst spät im Krankheitsverlauf gestellt. In den letzten Jahren haben sich durch die Etablierung neuer Untersuchungsmethoden wie der Gelenksonographie und der Kernspintomographie die Möglichkeiten zur früheren Erkennung einer Psoriasisarthritis deutlich verbessert. Dieses ist vor allem deswegen relevant, weil neue Medikamente wie Leflunomid und TNF$\alpha$-Blocker für die Therapie der Psoriasisarthritis zur Verfügung stehen. Vor allem die TNF- $\alpha$-Blocker induzieren deutlich höhere Remissionsraten als die bislang eingesetzten Substanzen erlauben. Die Entwicklung weiterer Substanzen, die selektiv die immunologischen Abläufe bei der Psoriasisarthritis beeinflussen, wird in Zukunft neue therapeutische Möglichkeiten eröffnen.
Abstract

Psoriatic arthritis is often diagnosed late in the course of the disease. Earlier diagnosis has been facilitated by novel diagnostic tools such as joint sonography and magnetic resonance imaging. In addition, novel agents such as leflunomide and TNF- $\alpha$-inhibitors have been introduced which allow more efficient therapy. In the future, new therapeutic opportunities will be offered by drugs that allow selective interference with the pathophysiological processes occurring in psoriatic arthritis.

\section{Definition, Epidemiologie und Ätiopathogenese}

Die Psoriasisarthritis (PsA) wird nach Moll und Wright [1] als seronegative Spondylarthritis (Befall peripherer Gelenke und des Stammskelettes), die mit einer Psoriasis assoziiert ist, definiert. Es werden verschiedene Varianten abgegrenzt (Tab.1), die die Vielfältigkeit der klinischen Erscheinungsbilder reflektieren.

Die Psoriasis weist in Mitteleuropa eine Prävalenz von etwa $1-3 \%$ auf. Ihr Verlauf wird in bis zu 30\% der Fälle durch eine Ar- thritis kompliziert, wobei die Häufigkeitsangaben in der Literatur erheblich schwanken [2,3]. Während die Psoriasisarthritis Männer und Frauen etwa gleich häufig betrifft, überwiegt beim Befall des Achsenskeletts das männliche Geschlecht deutlich.

Die Ätiopathogenese der Psoriasisarthritis ist weitgehend ungeklärt. Familien- und Zwillingsuntersuchungen verweisen auf eine genetische Komponente. HLA-B27 wurde gehäuft in Assoziation mit einer Psoriasisarthritis des Achsenskeletts gefunden während HLA-B39 eine Beziehung zur polyarthritischen Ver-

${ }^{1}$ Schwerpunkt Rheumatologie und Klinische Immunologie der Medizinischen Klinik und Poliklinik II

der Universität Würzburg

2 Klinik für Dermatologie, Venerologie und Allergologie, Universitätsklinikum Mannheim,

Fakultät für Klinische Medizin Mannheim der Ruprecht-Karls-Universität Heidelberg

Korrespondenzadresse

PD Dr. Christian Kneitz · Schwerpunkt Rheumatologie und Klinische Immunologie

der Medizinischen Klinik und Poliklinik II · Universität Würzburg · Klinikstr. 6 · 97070 Würzburg ·

E-mail: Kneitz_C@klinik.uni-wuerzburg.de

Bibliografie

Akt Dermatol 2006; 32: 216-220 @ Georg Thieme Verlag KG Stuttgart • New York

DOI 10.1055/s-2006-925305 · ISSN 0340-2541 
Tab. 1 Klassifikation der Psoriasisarthritis nach Moll und Wright

\begin{tabular}{|c|c|c|}
\hline Form & Häufigkeit & Kennzeichen, Besonderheiten \\
\hline $\begin{array}{l}\text { Oligoarthritis bzw. } \\
\text { asymmetrische } \\
\text { Form }\end{array}$ & $>50 \%$ & $\begin{array}{l}\text { milder Verlauf, Befall weniger Gelenke, } \\
\text { mitunter im Strahl („Wurstfinger und } \\
\text {-zehen“) }\end{array}$ \\
\hline $\begin{array}{l}\text { Symmetrische } \\
\text { Polyarthritis }\end{array}$ & $15-35 \%$ & $\begin{array}{l}\text { von rheumatoider Arthritis oft kaum zu } \\
\text { unterscheiden }\end{array}$ \\
\hline $\begin{array}{l}\text { Distale interpha- } \\
\text { langeale (DIP) } \\
\text { Arthritis }\end{array}$ & $5-15 \%$ & $\begin{array}{l}\text { oft einseitiger Befall weniger Gelenke, } \\
\text { häufig Nagelbeteiligung }\end{array}$ \\
\hline $\begin{array}{l}\text { Spondylarthritis } \\
\text { (Spondylitis) }\end{array}$ & $\begin{array}{l}\text { ca. } 5 \% \text { (mit } \\
\text { Arthritis: } \\
10-15 \%)\end{array}$ & typischer Befall des Stammskelettes \\
\hline Arthritis mutilans & $<5 \%$ & $\begin{array}{l}\text { ungünstiger Verlauf mit schweren De } \\
\text { formitäten und Bewegungseinschrän- } \\
\text { kungen, oft ausgeprägte Hautbeteili- } \\
\text { gung, reduzierter Allgemeinzustand }\end{array}$ \\
\hline
\end{tabular}

laufsform aufzuweisen scheint [5]. Auch exogene Faktoren, wie z. B. Streptokokkeninfektionen, sind in der Lage, eine Psoriasis zu triggern. HIV-Infizierte zeigen eine höhere Prävalenz für das Auftreten einer Psoriasisarthritis.

Wenngleich die von den meisten Autoren postulierte zentrale Rolle der T-Lymphozyten für die Pathogenese der psoriatischen Hautläsionen nicht unumstritten ist, kann doch davon ausgegangen werden, dass die das synoviale Kompartiment infiltrierenden CD8+ T-Zellen von wesentlicher Bedeutung für den arthritischen Prozess sind. Relevante (Auto-)Antigene sind bislang allerdings nicht identifiziert worden. Ähnlich wie bei der rheumatoiden Arthritis konnte die Bedeutung von proinflammatorischen Zytokinen wie TNF- $\alpha$ für die Pathogenese der Erkrankung belegt werden [4].

\section{Klinische Merkmale und Diagnose}

In der Mehrzahl der Fälle (etwa 75\%) gehen die Hautmanifestationen der Psoriasis der Gelenkaffektion viele Jahre voraus. Seltener findet sich ein synchrones Auftreten von Haut- und Gelenk- beteiligung bzw. eine Arthropathie ohne Nachweis von Hautläsionen (Psoriasisarthritis sine psoriase). Die Frage, ob das Ausmaß der Hautveränderungen, wie es sich z. B. im PASI-Score reflektiert, mit der Schwere der Arthritis korreliert, wird auch heute noch kontrovers diskutiert. Dieses liegt nicht zuletzt darin begründet, dass die Symptome der Gelenkerkrankung anfangs oft nur gering ausgeprägt sind, so dass die diagnostische Einordnung schwierig sein kann. Während bei einem großen Teil der Patienten die eher milde, oligoartikuläre Verlaufsform überwiegt (Abb.1), entwickeln über 30\% der Patienten einen schweren, polyartikulären Verlauf (Abb. 2), der mit einer erheblichen Beeinträchtigung der Gelenkfunktion einhergehen kann. Obwohl vorwiegend die Gelenke der Finger und Zehen betroffen sind, kommt auch ein Befall der Knie- und Sprunggelenke vor. Die Erkrankung kann sich als periphere Polyarthritis mit meist symmetrischem Gelenkbefallsmuster (bis zu 35\%) manifestieren und ist dann, besonders bei fehlender Hautbeteiligung, differenzialdiagnostisch schwer von der rheumatoiden Arthritis abzugrenzen. Die häufigere, asymmetrische Oligoarthritis (>50\%) der distalen und proximalen Interphalangealgelenke und Metakarpalgelenke ist oft begleitet von einer digitalen Tendosynovitis, die klinisch als Daktylitis mit charakteristischen „Wurstfingern“ (,sausage digits“) imponiert (Abb.1). Bei mutilierendem Verlauf (5\%) kann es unter anderem zur Entwicklung sogenannter „Teleskop-Finger“ kommen. Weitere Manifestationsformen sind die spinalen Arthritiden in Form einer asymmetrischen Spondylitis oder Sakroileitis (bis 15\%). Die gebräuchlichste Einteilung nach Moll und Wright (Tab.1) versucht den aufgeführten klinischen Verlaufsformen Rechnung zu tragen, wobei sich die einzelnen Formen oft überlappen [1].

Als extraartikuläre Symptome werden schmerzhafte Enthesiopathien und Tendosynovitiden sowie selten eine Iritis gefunden. Eine psoriatische Nagelbeteiligung wird häufig diagnostiziert und kann bei sonst fehlenden Hautmanifestationen wichtige Hinweise für die differenzialdiagnostische Einordnung der Arthritis liefern. Umgekehrt zeigen mehr als dreiviertel aller Patienten mit Psoriasisarthritis charakteristische Veränderungen an den Fingernägeln.

Laborchemisch können sich als unspezifische Aktivitätszeichen der Psoriasisarthritis eine Erhöhung von BSG und Akute-Phase-

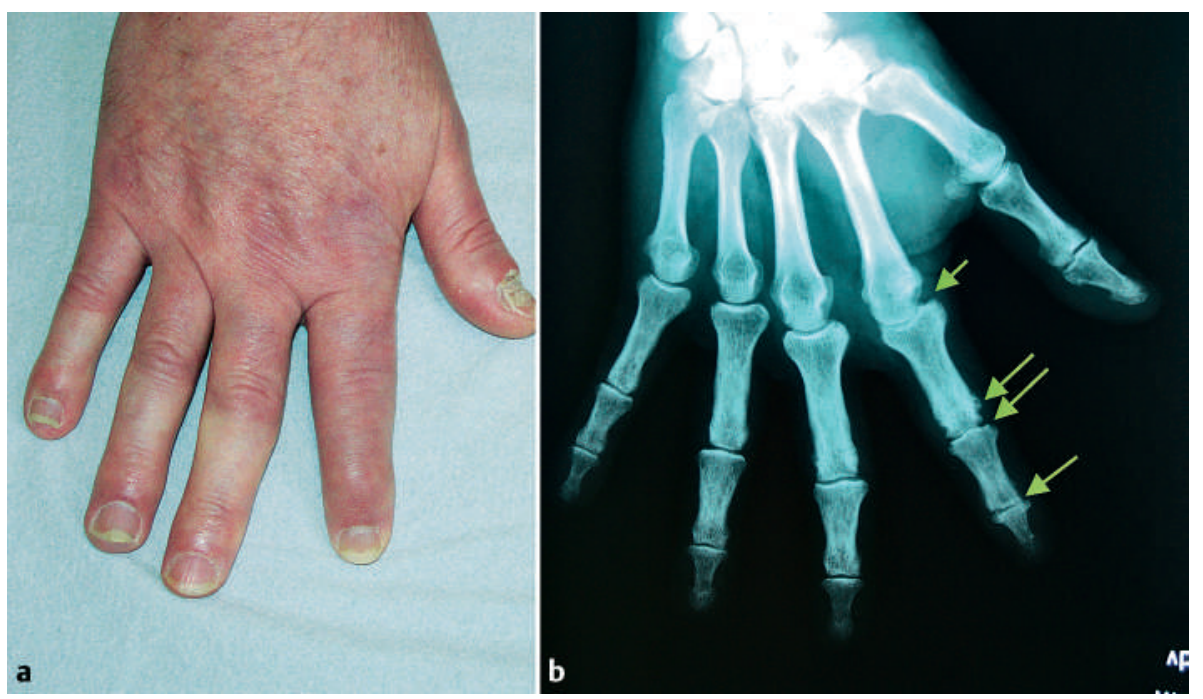

Abb. 1 Daktylitis bei einem Patienten mit oligoarthritischer Verlaufsform der Psoriasisarthritis (Dig. II re, a). Im Röntgenbild erkennt man ein charakteristisches Nebeneinander destruierender und proliferativer Veränderungen der Gelenk- bzw. Knochenstrukturen (b, Pfeile). 

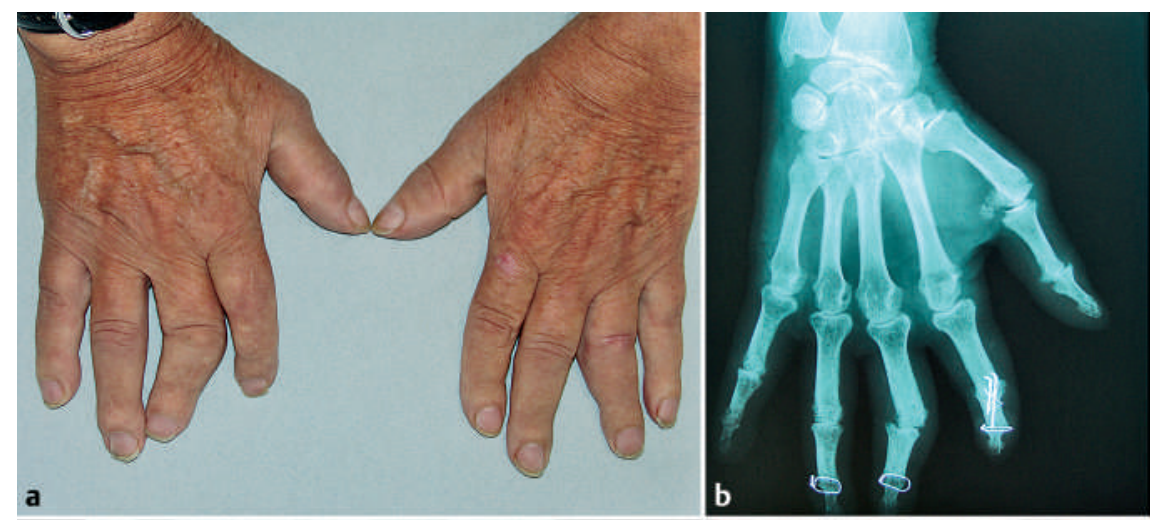

Abb. 2 Mutilierende Verlaufsform der Psoriasisarthritis mit ausgeprägten Destruktionen an Händen (a, b) und Vorfüßen (c-e).
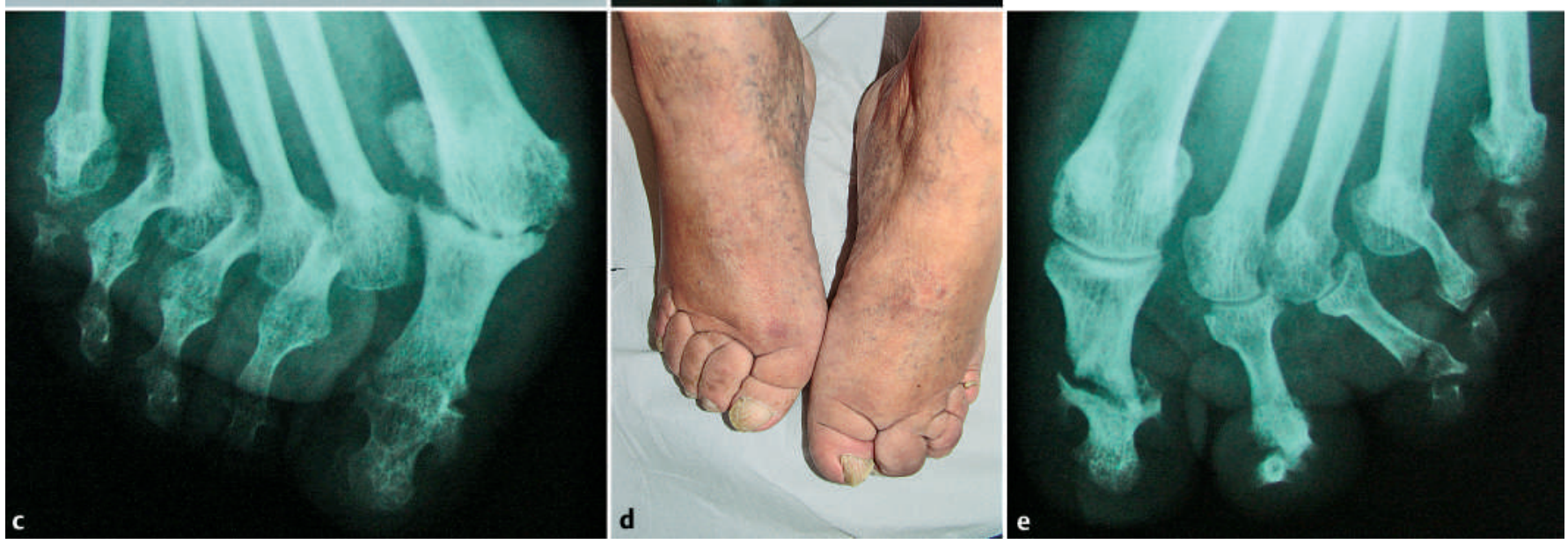

Proteinen (CRP) finden. Die Harnsäure ist nicht selten erhöht. Typischerweise liefert die Bestimmung des Rheumafaktors, von CCP- und antinukleären Antikörper negative Befunde. Der Nachweis der genannten Parameter sollte zu differenzialdiagnostischen Überlegungen anregen, schließt aber das Vorliegen einer Psoriasisarthritis keinesfalls aus. Der Nachweis distinkter HLAAntigene, die als krankheitsdisponierende Faktoren identifiziert wurden, ist in der Praxis von untergeordneter Bedeutung.

Radiologisch charakteristisch sind das vertikale und horizontale Gelenkbefallsmuster von Fingern und Zehen mit gleichzeitigem Vorkommen von Knochenproliferation und -destruktion an distalen und proximalen Interphalangealgelenken (Abb.1 u.2). Veränderungen an Synchondrosen und Sehnenansätzen sowie bei schweren Verläufen auch Gelenkmutilationen (z.B. „pencil in cup“) oder Ankylosen sind neben der Sakroileitis weitere radiologisch dokumentierbare Befunde der Psoriasisarthritis. Die konventionelle Bildgebung durch Röntgenaufnahmen kann durch die Kernspintomographie ergänzt werden, die besonders zum Nachweis von Enthesitiden oder einer diskreten Synovialitis wichtige diagnostische Hinweise liefern kann. Für den Nachweis von Enthesitiden eignet sich auch die Sonographie. Die Durchführung einer Skelettszintigraphie kann in unklaren Fällen helfen, das Ausbreitungsmuster zu bestimmen und dient dann vor allem zur differenzialdiagnostischen Abgrenzung von degenerativen Gelenkveränderungen. Die Skelettszintigraphie zeigt gerade bei der Psoriasisarthritis eine hohe Sensitivität bezüglich des Nachweises der Krankheitsaktivität. Sie lässt jedoch keine Artdiagnose zu, die zur Differenzierung der verschiedenen entzündlichen Gelenkerkrankungen beitragen könnte.
Differenzialdiagnostisch ist die Psoriasisarthritis von anderen Spondylarthropathien abzugrenzen. Hier ist das SAPHO-Syndrom (Synovitis-Akne-Pustulose-Hyperostose, Osteitis-Syndrom) zu nennen, bei dem betroffene Patienten $u$. a. neben einer palmoplantaren Pustulose Gelenkaffektionen in der Sternocostoclavicularregion zeigen. Bei Befall des Stammskelettes und gleichzeitigem Nachweis von HLA-B27 kann die Abgrenzung eines M. Bechterew, besonders bei Fehlen typischer Hautmanifestationen, schwierig sein. Gleiches gilt bei Befall einzelner großer Gelenke für die reaktive Arthritis, auf deren Vorliegen ein vorausgegangener Infekt des Respirations-, Gastrointestinal- oder Urogenitaltraktes hinweisen kann. Für eine enteropathieassoziierte Arthritis spricht eine gastrointestinale Symptomatik. Die rheumatoide Arthritis ist durch ein symmetrisches Gelenkbefallsmuster mit transversaler Gelenkbeteiligung, das Fehlen des Befalls der distalen Interphalangealgelenke und den positiven Nachweis von Rheumafaktor und CCP-Antikörpern sowie anhand typischer radiologisch nachweisbarer Veränderungen abgrenzbar. Gerade im Initialstadium der Erkrankungen gelingt eine eindeutige Zuordnung zu einer der genannten Krankheitsentitäten mitunter nicht, so dass dann erst der weitere Krankheitsverlauf Klärung bringt.

\section{Therapie}

Mehr als die Hälfte aller Patienten mit Psoriasisarthritis weisen nach 10 Jahren ausgeprägte Gelenkzerstörungen in mehreren Lokalisationen auf. Besonders bei Patienten mit polyartikulärem Befallsmuster (ca. 30\%) lässt sich eine rasche Progredienz beobachten. Dabei ist davon auszugehen, dass, ähnlich wie bei der 
rheumatoiden Arthritis, bereits zu Beginn der Erkrankung eine Zerstörung der Gelenke in relevantem Umfang eintritt [7]. Es ist daher nicht verwunderlich, dass sich trotz der Unterschiede in der Pathogenese beider Erkrankungen die Konzepte der medikamentösen Therapie ähneln. Bei der Psoriasisarthritis ist allerdings zu beachten, dass die medikamentöse Therapie auch unter Berücksichtigung der psoriatischen Hautveränderungen erfolgen sollte und daher eine enge Zusammenarbeit von Dermatologen und Rheumatologen anzustreben ist.

Konventionelle nichtsteroidale Antirheumatika (NSAR) und die neueren Cyclooxygenase-2-Inhibitoren (im Folgenden als „Coxibe“ bezeichnet) eignen sich aufgrund ihrer guten analgetischen und antiphlogistischen Eigenschaften zur symptomatischen Therapie von Arthritiden und Enthesitiden. Dabei zeichnen sich Coxibe durch ein im Vergleich zu konventionellen NSAR geringeres Risiko für die Entstehung einer klinisch relevanten gastrointestinalen Blutung aus, was gleichzeitig bedeutet, dass ein nicht zu vernachlässigendes Risiko verbleibt. Alternativ kann auch die gleichzeitige Gabe eines Protonenpumpenblockers die gastrointestinale Toxizität von konventionellen NSAR vermindern. Zahlreiche weitere Nebenwirkungen und Kontraindikation der Substanzen sind zu beachten. Hinsichtlich der Coxibe, möglicherweise auch der NSAR, besteht ein erhöhtes Risiko für schwere kardiovaskuläre Nebenwirkungen und Hautreaktionen (siehe auch homepage der EMEA (http://www.emea.eu.int/), so dass die Substanzen nur nach besonderer Risikoabwägung eingesetzt werden sollten. Weiterhin ist anzumerken, dass NSAR oder Coxibe bislang in keiner größeren kontrollierten Studie zur Therapie der Psoriasisarthritis überprüft wurden.

In vielen Fällen sind die rein symptomorientiert eingesetzten NSAR nicht in der Lage, den Krankheitsverlauf ausreichend zu kontrollieren. Während Kortikosteroide bei der rheumatoiden Arthritis probate Mittel zur Überbrückung der Zeit bis zum Einsetzen der Wirkung einer Basistherapie darstellen bzw. Schübe der Erkrankung abfangen können, so ist ihr Einsatz bei der Psoriasisarthritis kritischer einzuschätzen. Dieses gilt besonders hinsichtlich des Aufflammens psoriatischer Hauterscheinungen nach Dosisreduktion [8]. Bei schweren, rasch progredienten Krankheitsverläufen kann der Einsatz systemischer Glukokortikoide allerdings dazu beitragen, eine Kontrolle der Arthritis zu erzielen, bis die in solchen Fällen einzuleitende Basistherapie greift.

Als wirksame Basistherapeutika zur Behandlung der Psoriasisarthritis gelten Sulfasalazin, Methotrexat und Cyclosporin A. Allerdings liegen für diese Substanzen keine kontrollierten Studien vor, die eine Hemmung der für den Krankheitsverlauf und die Prognose besonders wichtigen radiologischen Progression dokumentieren [9]. Grundsätzlich ist zu beachten, dass sowohl für die Therapie der Psoriasis wie auch der Psoriasisarthritis nicht alle hier besprochenen Substanzen eine Zulassung besitzen (siehe Tab. 2)

Sulfasalazin (2-3 g/Tag) wird eher bei mildem, Methotrexat (bis $20 \mathrm{mg} /$ Woche) und Cyclosporin A (Dosierungen 2,5 mg/kg Körpergewicht) eher bei aggressivem und mit Sulfasalazin nicht beeinflussbarem Krankheitsverlauf, sowie dann, wenn die Hautbeteiligung im Vordergrund steht, verwendet. Der Einsatz von Methotrexat wird oft durch gastrointestinale, hepatische sowie hä-
Tab. 2 Aktueller Zulassungsstatus von Wirkstoffen zur Behandlung der Psoriasis/Psoriasisarthritis (in Deutschland)

\begin{tabular}{lll}
\hline Präparat & Psoriasis & Psoriasisarthritis \\
\hline Hydroxychloroquin & nein & Nein \\
\hline Sulfasalazin & nein & Nein \\
\hline Gold [i.m.] & nein & ja \\
\hline Methotrexat & ja & ja \\
\hline Cyclosporin A & ja & nein \\
\hline Leflunomid & nein & ja \\
\hline Etanercept & ja & ja \\
\hline Infliximab & ja & ja \\
\hline Adalimumab & nein & ja \\
\hline
\end{tabular}

matologische Nebenwirkungen, der von Cyclosporin A besonders durch die renale Toxizität der Substanz limitiert. Ein engmaschiges klinisches und laborchemisches Monitoring der Patienten ist zur rechtzeitigen Erkennung von Therapienebenwirkungen unabdingbar. Antimalariamittel und Gold erscheinen nicht zuletzt aufgrund der bekannten kutanen Nebenwirkungen für die Behandlung der Psoriasisarthritis weniger gut geeignet. Der Einsatz neuerer Immunsuppressiva wie Tacrolimus (FK506), oder Mykophenolat-Mofetil zeigte an kleinen Patientenkollektiven einen günstigen Einfluss auf die Psoriasisarthritis. Ihre Anwendung sollte zunächst kontrollierten Studien vorbehalten bleiben. Die Wirksamkeit von Leflunomid auf Gelenke und Haut wurde bei Patienten mit Psoriasisarthritis in einer Plazebo-kontrollierten Doppelblind-Studie gezeigt, so dass Leflunomid in Deutschland zur Behandlung der Psoriasis-Arthritis zugelassen wurde [10].

Die Einführung der Biologicals erweiterte die therapeutischen Möglichkeiten bei entzündlich-rheumatischen Erkrankungen erheblich. Biologicals bilden eine Gruppe von Wirkstoffen aus genetisch modifizierten Eiweißmolekülen, die als monoklonale Antikörper oder lösliche Rezeptorkonstrukte meist gegen Zytokine oder zelluläre Oberflächenstrukturen gerichtet sind. Biologicals sind in der Lage, selektiv in Entzündungsreaktionen einzugreifen und beispielsweise die Wirkung proinflammatorischer Zytokine (TNF- $\alpha$, IL-1, IL-6) zu hemmen oder durch die Blockade bestimmter Rezeptoren (CD28, CD40) die Stimulation pathogenetisch relevanter Zellen zu blockieren. Gerade die Blockade von TNF- $\alpha$ hat sich in der Therapie der rheumatoiden Arthritis als sehr erfolgreich erwiesen, so dass die TNF- $\alpha$-Blocker Adalimumab, Etanercept und Infliximab schon seit mehreren Jahren bei dieser Indikation erfolgreich eingesetzt werden. Nachdem die Wirksamkeit dieses Behandlungskonzeptes auch für die Psoriasisarthritis überzeugend belegt werden konnte, sind TNF- $\alpha$-Hemmer auch für diese Indikation zugelassen worden [11 - 13]. Besonders hervorzuheben ist, dass neben einer Verbesserung der Lebensqualität, rückläufigen Arthritiden und damit verbesserter Gelenkfunktion in vielen Fällen auch die radiologische Progression der Psoriasisarthritis aufgehalten [11,13] und eine Besserung der psoriatischen Hauterscheinungen erreicht werden kann. Ähnlich wie bei der rheumatoiden Arthritis gibt es Hinweise, dass bei ungenügendem Ansprechen auf die Therapie ein Wechsel von einem zu einem anderen TNF- $\alpha$-Blocker sinnvoll sein kann, um das therapeutische Ansprechen zu verbessern [14]. Ebenso sollte 
in therapierefraktären Fällen eine Kombinationstherapie von TNF- $\alpha$-Blockern mit Methotrexat erwogen werden.

Für andere Kombinationstherapien liegen nur wenige Erfahrungen vor, so dass solche nur unter strenger Überwachung durchgeführt werden sollten. Auch ohne Vorliegen einer Psoriasisarthritis können sowohl Etanercept wie auch Infliximab zur Therapie der mittelschweren bis schweren Plaque-Psoriasis eingesetzt werden, sofern zuvor systemische Standardtherapien versagt haben. Eine interessante Frage ist, ob der Einsatz von TNF- $\alpha$-Blockern zur Therapie der schweren Psoriasis geeignet ist in dieser Patientengruppe die Entwicklung einer Gelenkbeteiligung, die in der Regel erst nach Jahren auf die Hautmanifestation folgt, zu verhindern.

Kontraindikationen für den Einsatz von TNF- $\alpha$-Inhibitoren sind schwere Herzinsuffizienz (ab NYHA Grad III), chronische Infektionen und Malignome; auf Lebendimpfungen sollte unter TNF$\alpha$-Blockade verzichtet werden. Da unter TNF- $\alpha$-Hemmung eine Tuberkulose reaktiviert werden kann, ist eine solche vor Therapiebeginn auszuschließen. Gemäß den Empfehlungen des PaulEhrlich-Instituts bzw. der Deutschen Gesellschaft für Rheumatologie muss vor der Erstgabe ein standardisiertes Screening erfolgen. Dieses umfasst eine strukturierte Anamnese, eine klinische Untersuchung, einen PPD-Tuberkulintest nach Mendel-Mantoux und eine Röntgenuntersuchung des Thorax. Das hier skizzierte TBC-Screening wird für alle zugelassenen TNF- $\alpha$-Blocker gleichermaßen empfohlen. Bei Nachweis einer aktiven Tuberkulose sind TNF- $\alpha$-Blocker absolut kontraindiziert. Ergibt sich der Nachweis einer latenten Tuberkulose (anamnestisch, radiologisch und/oder im Tuberkulin-Test Hinweis auf stattgehabte Infektion), so kann erwogen werden, die Behandlung unter einer prophylaktischen Gabe von Isoniazid durchzuführen. Diese sollte vier Wochen vor der Therapie beginnen und über mindestens neun Monate durchgeführt werden. Die systemische Hemmung von TNF- $\alpha$ bewirkt eine signifikante Immunsuppression. Es muss daher mit einer erhöhten Anfälligkeit für schwer verlaufende Infektionen gerechnet werden, was bei der Betreuung der Patienten zu beachten ist. Daraus folgt, dass bei Vorliegen einer schweren Infektion eine Behandlung mit TNF- $\alpha$-Blockern unterbrochen bzw. erst gar nicht begonnen werden sollte. Unter der subkutanen Anwendung von Adalimumab und Etanercept können zumeist passagere Hautreaktionen auftreten, während bei der intravenösen Gabe von Infliximab in seltenen Fällen Infusionsreaktionen beobachtet werden. Generell stellt die TNF$\alpha$-Hemmung einen starken Eingriff in das Immunsystem dar und sollte nur von Ärzten, die eine große Erfahrung mit immunsuppressiven Therapien besitzen, durchgeführt werden.

Durch ein besseres Verständnis der der Psoriasisarthritis zugrunde liegenden immunologischen Abläufe gelang es somit, neue Behandlungskonzepte zu entwickeln, die geeignet sind, eine rasche Besserung der Gelenkproblematik sowie des Allgemeinbefindens herbeizuführen $[15,16]$. Es erscheint notwendig, über eine bessere Definition prognostisch relevanter Parameter (oligo- versus polyartikulärer Befall, niedrige versus hohe entzündliche Aktivität, Ausmaß der Hautbeteiligung, u. a.) frühzeitig Pa- tienten mit einem ungünstigen Krankheitsverlauf zu ermitteln, um diese früher und effektiver zu behandeln. Die Entwicklung und Einführung neuer Substanzen lässt hoffen, dass sich auch für bislang therapierefraktäre oder ungenügend ansprechende Patienten neue therapeutische Optionen eröffnen. Die enge interdisziplinäre Zusammenarbeit zwischen Dermatologen und Rheumatologen wird bei Patienten mit Psoriasisarthritis auch in Zukunft die Basis für die Entwicklung individuell optimierter Behandlungspläne darstellen.

\section{Danksagung}

Wir bedanken uns beim Institut für Röntgendiagnostik (Direktor: Prof. Dr. D. Hahn) für die Bereitstellung der Röntgenbilder.

\section{Literatur}

${ }^{1}$ Moll JM, Wright V. Psoriatic arthritis. Semin Arthritis Rheum. 1973; 3(1): $55-78$

${ }^{2}$ Gladman DD, Antoni C, Mease P, Clegg DO, Nash P. Psoriatic Arthritis: epidemiology, clinical features, course and outcome. Ann Rheum Dis 2005; 64 Suppl 2: ii $14-17$

${ }^{3}$ Leonard DG, O'Duffy JD, Rogers RS. Prospective analysis of psoriatic arthritis in patients hospitalized for psoriasis. Mayo Clin Proc 1978; 53 : $511-518$

${ }^{4}$ Ritchin C, Haas-Smith SA, Hicks D, Cappuccio J, Osterland CK, Looney RJ. Patterns of cytokine production in psoriatic synovium. J Rheumatol 1998; 25: $1544-1552$

${ }^{5}$ Gladman DD, Farewell VT, Kopciuk HA, Cook RJ. HLA antigens and progression in psoriatic arthritis. J Rheumatol 1998; 25: 730-733

${ }^{6}$ Gladman DD, Anhorn KA, Schachter RK, Mervart H. HLA antigens in psoriatic arthritis. J Rheumatol 1986; 13: 586-592

${ }^{7}$ Kane D, Stafford L, Bresnihan B, FitzGerald O. A prospective, clinical and radiological study of early psoriatic arthritis: an early synovitis clinic experience. Rheumatology 2003; 42: 1460-1468

${ }^{8}$ Baker H, Ryan TJ. Generalized pustular psoriasis. A clinical and epidemiological study of 104 cases. Br J Dermatol 1968; 80: 771 - 793

${ }^{9}$ Nash P, Clegg DO. Psoriatic arthritis therapy: NSAIDs and traditional DMARDs. Ann Rheum Dis 2005; 64: $74-77$

${ }^{10}$ Kaltwasser JP, Nash P, Gladman D, Rosen CF, Behrens F, Jones P, Wollenhaupt J, Falk FG, Mease P. Efficacy and safety of leflunomide in the treatment of psoriatic arthritis and psoriasis: a multinational, doubleblind, randomized, placebo-controlled clinical trial. Arthritis Rheum 2004; 50: 1939-1950

${ }^{11}$ Mease PJ, Kivitz AJ, Burch FX, Siegel EL, Cohen SB, Ory P, Salonen D, Rubenstein J, Sharp JT, Tsuji W. Etanercept treatment of psoriatic arthritis: safety, efficacy, and effect on disease progression. Arthritis Rheum 2004; 50: 2264-2272

12 Antoni C, Krueger GG, de Vlam K, Birbara C, Beutler A, Guzzo C, Zhou B, Dooley LT, Kavanaugh A. Infliximab improves signs and symptoms of psoriatic arthritis: results of the IMPACT 2 trial. Ann Rheum Dis 2005; 64: $1150-1157$

${ }^{13}$ Mease PJ, Gladman DD, Ritchlin CT, Ruderman EM, Steinfeld SD, Choy EH, Sharp JT, Ory PA, Perdok RJ, Weinberg MA. Adalimumab for the treatment of patients with moderately to severely active psoriatic arthritis: results of a double-blind, randomized, placebo-controlled trial. Arthritis Rheum 2005; 52: 3279-3289

${ }^{14}$ Strober BE. Successful treatment of psoriasis and psoriatic arthritis with etanercept and methotrexate in a patient newly unresponsive to infliximab. Arch Dermatol 2004; 140: 366

${ }^{15}$ Mease PJ. Psoriatic arthritis therapy advances. Curr Opin Rheumatol 2005; 17: 426-432

${ }^{16}$ Mease PJ, Antoni CE. Psoriatic arthritis treatment: biological response modifiers. Ann Rheum Dis 2005; 64 Suppl 2: ii78 - 82 